\title{
Calculation Tool for Optimal Wireless Design and Minimal Installation Cost of Indoor Wireless LANs
}

\author{
D. Plets, N. Machtelinckx, K. Vanhecke, J. Van Ooteghem, K. Casier, M. Pickavet, W. Joseph, L. Martens \\ Information Technology Department, Ghent University/iMinds \\ Gaston Crommenlaan 8, B-9050 Ghent, Belgium \\ david.plets@intec.ugent.be
}

\begin{abstract}
An algorithm is presented for the optimal placement of access points, followed by a minimization of the cost for connecting WLAN networks to the power and ethernet network. The algorithm is described and applied to a simple building layout. The algorithm outputs an overview of the different costs and allows WLAN installers to build the cheapest solution.
\end{abstract}

\section{INTRODUCTION}

In recent years, the use of wireless communication has increased drastically. Although deploying a wireless network obviously requires less cabling than deploying a wired network, the installation cost of wireless networks may still be considerable. Although many wireless network planner (algorithms) are available (e.g., [1], [2], [3]), installation cost has not been accounted for yet in the network planning phase. To the author's knowledge, this paper is the first to present a calculation tool for an optimal wireless design with a minimal installation cost of Wireless Local Area Networks (WLANs). The algorithm is applied to a simple building layout.

\section{Cost Minimization Algorithm}

\section{A. Configuration}

The algorithm for optimal network planning and minimal cabling cost will be illustrated for the simple building layout depicted in Fig. 1. It consists of two rooms sharing a metal wall. The locations of the power connection points (PCPs) and ethernet connection points (ECPs) are indicated with green and red dots respectively.

\section{B. Optimal network planning}

For the considered building and starting from an empty ground plan, the WiCa Heuristic Indoor Propagation Prediction (WHIPP) algorithm plans the wireless network in order to obtain a coverage rate of $100 \%$, according to the optimization algorithm described in [1]. The WHIPP algorithm is a heuristic planning algorithm, developed and validated for the prediction and optimization of wireless coverage in indoor environments. It takes into account the effect of the environment on the wireless propagation channel and bases its calculations on the determination of the dominant path between transmitter and receiver, i.e., the path along which the signal encounters the lowest obstruction. This path is determined with a multidimensional optimization algorithm that searches the lowest total path loss, consisting of a distance loss (accounting for the length of the propagation path), a cumulated wall loss (accounting for the walls penetrated along the propagation path), and an interaction loss (accounting for the propagation direction changes of the path, e.g., diffraction around corners). The model, constructed for the $2.4-2.6 \mathrm{GHz}$ band, has shown excellent correspondence between predictions and validation measurements [1]. The network planning algorithm is based on the consecutive selection of the best next AP from a pool of possible APs at different locations. A further optimization is applied by merging two APs where possible (without reducing coverage). For the considered configuration, the algorithm outputs a WiFi network with 2 APs (due to the metal wall) with an Equivalent Isotropically Radiated Power (EIRP) of $16 \mathrm{dBm}$, (see purple dot in Fig. 1). In a next phase, the installation cost will be minimized for this AP configuration.

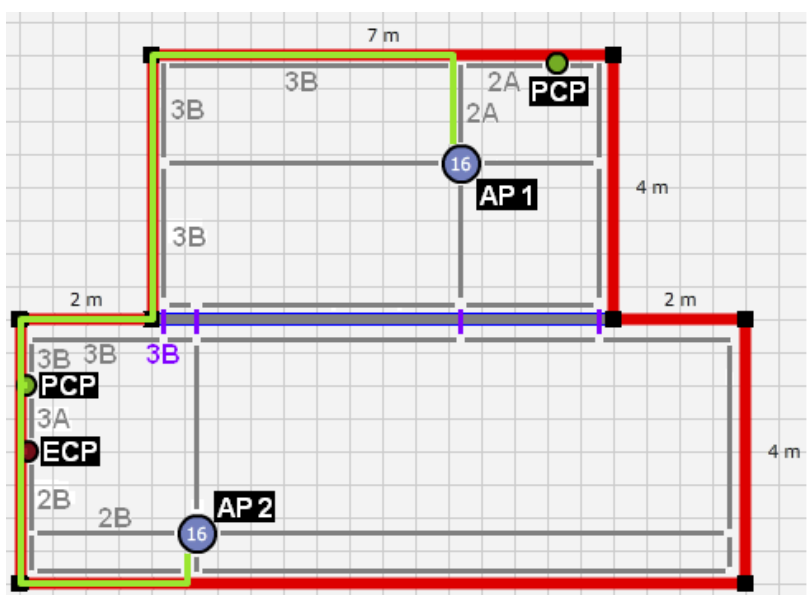

Fig. 1. Ground plan with reference to algorithm steps (adding cable gutters 2A-2B in step 2, and 3A-3B in step 3). EIRP [dBm] of AP indicated inside purple dot; red wall = brick; grey wall = metal; grey graph edges connect AP nodes and/or wall nodes; purple graph edges represent drilled holes; $\mathrm{AP}=$ access point $\mathrm{PCP}=$ power connection point $\mathrm{ECP}=$ ethernet connection point; light green lines $=$ final cabling)

\section{Minimization of installation cost}

Access points only function if they are connected to both a PCP and ECP. The cabling cost minimization algorithm optimizes the location and the amount of power and ethernet cables that are needed to connect each AP in the considered indoor environment to both a PCP and an ECP with the lowest possible cost, based on the algorithm's input data: the AP, 
PCP, and ECP positions, the cost of cable (ethernet and power) and cable gutter (containing the cables) per meter, drilling holes through walls (material-dependent), and working hours. The number of working hours is determined by the number of holes to be drilled, the number of meters of cables and cable gutters to be installed (dependent on material of wall they are attached to), and the number of APs to be installed. It is clear that the physical layout of the ground plan will greatly influence the output of the algorithm.

1) Algorithm: The cost minimization algorithm is based on graph theory. Therefore the plan is converted to a graph, where the graph nodes are APs, PCPs, ECPs, or points on a wall inside a room. The graph edges represent possible connections between two nodes. These are a cable gutter position (grey edges in Fig. 1 between AP nodes and/or wall nodes in the same room) or a hole in a wall, i.e., two nodes with the same coordinates, but in different rooms (purple edges in Fig. 1). Each edge is attributed a weight equal to the cost of installing the connection (cabling installation or drilling cost).

To find the solution with the minimal cost, an adapted version of the Kruskal [4] algorithm for constructing a Minimum Spanning Tree is applied to the created graph. The MST does not have to be fully connected though, only the AP nodes need a connection with a PCP and an ECP. The algorithm consists of the following steps:

1) Calculate shortest (cheapest) path between each two nodes of the graph with Dijkstra algorithm

2) Add cheapest cable gutter that connects one of the APs with a PCP, ECP, or other AP. In our case, this is cable gutter 2A, connecting AP 1 to the upper PCP. Then, for the remaining APs: add the cheapest cable gutter that connects the AP with a PCP, ECP, another AP, or a cabled $^{1}$ graph node (here: cable gutter $2 \mathrm{~B}$, from AP 2 to the (only) ECP).

3) While not all APs are fully connected ${ }^{2}$ : add the cheapest cable gutter that connects either two tree components ${ }^{3}$ or either a tree component of the AP to a PCP or an ECP (whichever one is not yet cabled in the tree component). Here, AP 1 is not yet connected to an ECP and AP 2 is not yet connected to a PCP. Therefore, the algorithm connects the ECP to the lower PCP (cable gutter $3 \mathrm{~A}$ in Fig. 1), because it fully connects AP 2 with the lowest cost. To also have AP 1 fully connected, cable gutters $3 \mathrm{~B}$ need to be added, as well as a hole in the metal wall between the two rooms (purple edge 3B).

4) Update cable gutters, since adding new gutters can make others redundant. Here, the cable gutter $2 \mathrm{~A}$ along the wall in the upper room is removed: cable gutters $3 \mathrm{~B}$ are required anyway (to connect the AP to the $\mathrm{ECP}$ ) and the cost to install power cables along these gutters is lower

\footnotetext{
${ }^{1}$ cabled $=$ connected to a cable gutter

${ }^{2}$ fully connected AP $=$ AP that is connected to both a PCP and an ECP

${ }^{3}$ tree component $=$ partial set of the graph nodes, which are interconnected by cable gutters
}

than the sum of the cost of installing that cable gutter $2 \mathrm{~A}$ and installing a power cable along it.

5) For all APs: connect AP to PCP with power cable and to ECP with ethernet cable along the shortest path along the cable gutters. This yields the layout of Fig. 1. The location of the cable gutters is indicated in light green, and ethernet and power cables are installed along them, between the APs and the ECPs (PCPs).

\section{Evaluation}

The tool automatically outputs an overview of the installation costs, as listed in Table I. Unit prices can be modified by the user (different manufacturer, different worker), which may lead to different optimal cable positions. The total cost for the considered scenario equals $\$ 986.24$, whereas traditional network planners only take into account the AP cost (i.e., $\$ 230$, only $23 \%$ of the total cost).

TABLE I

COST OVERVIEW.

\begin{tabular}{lrrr} 
& & Unit price & Cost [\$] \\
\hline \hline Cable gutter & $20.02 \mathrm{~m}$ & $\$ 17.75 / \mathrm{m}$ & 355.36 \\
\hline Ethernet cable & $19.9 \mathrm{~m}$ & $\$ 2 / \mathrm{m}$ & 39.8 \\
\hline Power cable & $19.9 \mathrm{~m}$ & $\$ 6.2 / \mathrm{m}$ & 123.38 \\
\hline AP & 2 & $\$ 115$ & 230 \\
\hline Working hours & $2.53 \mathrm{~h}$ & $\$ 90 / \mathrm{h}$ & 227.7 \\
$\quad$ Installing APs & $1 \mathrm{~h}$ & & 90 \\
Making holes & $0.2 \mathrm{~h}$ & & 18 \\
Installing cabling & $1.33 \mathrm{~h}$ & & 119.7 \\
\hline Holes & 1 & $\$ 10$ & 10 \\
\hline Total cost & & & $\mathbf{9 8 6 . 2 4}$
\end{tabular}

\section{CONCLUSIONS}

An algorithm is presented for optimal wireless network planning and minimization of the installation cost of access point cabling. For a simple application case, it is shown that the installation (cables, gutters, drilling) costs more than 3 times as much as the APs themselves, indicating the necessity of cabling cost calculation during the network planning phase. Future research includes an economic optimization where the choice of the AP locations is also included in the cost minimization process.

\section{REFERENCES}

[1] D. Plets, W. Joseph, K. Vanhecke, E. Tanghe, and L. Martens, "Coverage Prediction and Optimization Algorithms for Indoor Environments," EURASIP Journal on Wireless Communications and Networking, Special Issue on Radio Propagation, Channel Modeling, and Wireless, Channel Simulation Tools for Heterogeneous Networking Evaluation, vol. 1, 2012. [Online]. Available: http://jwcn.eurasipjournals.com/content/2012/1/123

[2] R.P. Torres, L. Valle, M. Domingo, M.C. Diez, "CINDOOR: an engineering tool for planning and design of wireless systems in enclosed spaces," IEEE Antennas and Propagation Magazine, vol. 41, no. 4, pp. 11-22, Sept. 1999.

[3] Aerohive Networks, "Aerohive Wi-Fi Planning Tool," Website, http://www.aerohive.com/build-your-network.

[4] J. B. Kruskal, "On the Shortest Spanning Subtree of a Graph and the Traveling Salesman Problem," Proceedings of the American Mathematical Society, vol. 7, no. 1, pp. 48-50, February 1956. 